Europhys. Lett., 54 (4), pp. 449-455 (2001)

\title{
Enhancement of the electron screening effect for $d+d$ fusion reactions in metallic environments
}

\author{
K. Czerski, A. Huke, A. Biller, P. Heide, M. Hoeft and G. Ruprecht \\ Institut für Atomare und Analytische Physik, Technische Universität Berlin \\ Sekr. PN 3-1, D-10623 Berlin, Germany
}

(received 5 June 2000; accepted in final form 8 March 2001)

PACS. 25.45.Hi - Transfer reactions.

PACS. 95.30.-k - Fundamental aspects of astrophysics.

PACS. 95.30.Dr - Atomic processes and interactions.

\begin{abstract}
To study the electron screening of nuclear reactions in metallic environments, angular distributions and thick target yields of the fusion reactions ${ }^{2} \mathrm{H}(\mathrm{d}, \mathrm{p})^{3} \mathrm{H}$ and ${ }^{2} \mathrm{H}(\mathrm{d}, \mathrm{n})^{3} \mathrm{He}$ have been measured on deuterons implanted in three different metal targets ( $\mathrm{Al}, \mathrm{Zr}$ and $\mathrm{Ta}$ ) for beam energies ranging from 5 to $60 \mathrm{keV}$. The experimentally determined values of the screening energy are about one order of magnitude larger than the value achieved in a gas target experiment and significantly larger than the theoretical predictions. A clear target material dependence of the screening energy has been established.
\end{abstract}

Introduction. - At sufficiently low projectile energies an enhancement of the cross-section for charged-particle-induced nuclear reactions can be observed. This is due to the shielding of the charges of reacting nuclei by surrounding electrons which leads to an increase of the Coulomb barrier penetrability and enhances the measured cross-section in comparison to the bare nuclei case. This effect, known as electron screening, was originally discussed for the dense plasma in the interior of stars [1], where, due to screening, the nuclear-reaction rates can be increased by many orders of magnitude. For laboratory thermonuclear reactions, the screening effect was predicted [2] and experimentally verified for several light nuclear systems [3].

In the simplest picture, the enhancement of the cross-section results from the gain of electronic binding energy (called screening energy $U_{\mathrm{e}}$ ) which can be transferred to the relative motion of the colliding nuclei. In an adiabatic limit, i.e. with velocities $v_{\text {nuclear }} \ll v_{\text {electron }}$, this energy shift can be treated as constant. Consequently, the enhancement factor $f$ defined as the ratio between the cross-sections for screened and bare nuclei can be calculated as follows [2]:

$$
f(E)=\frac{\sigma_{\mathrm{scr}}}{\sigma_{\text {bare }}}=\frac{\frac{1}{E+U_{\mathrm{e}}} S\left(E+U_{\mathrm{e}}\right) e^{-2 \pi \eta\left(E+U_{\mathrm{e}}\right)}}{\frac{1}{E} S(E) e^{-2 \pi \eta(E)}},
$$

where the transformation of the cross-section to the astrophysical $S$-factor, a weak-energy dependent function, has been applied; $\eta$ is the Sommerfeld parameter and $E$ is the CMS (c) EDP Sciences 
energy. The enhancement factor $f$ is very close to unity for energies $E>100 U_{\mathrm{e}}$ (for light nuclear systems $U_{\mathrm{e}}$ is of order $100 \mathrm{eV}$ ) and increases exponentially for lower energies.

On the other hand, this energy shift can be easily calculated in the frame of the BornOppenheimer approximation. If the potential energy $W(r)$ between the colliding ions is known, the screening function $S_{\mathrm{c}}(r)$ may be obtained as a modification of the internuclear Coulomb potential and expanded around $r=0$

$$
W(r)=\frac{Z_{1} Z_{2} e^{2}}{r} S_{\mathrm{c}}(r)=\frac{Z_{1} Z_{2} e^{2}}{r}\left(1-\frac{r}{D_{\mathrm{s}}}+\cdots\right),
$$

where $D_{\mathrm{s}}$ is the short-range screening distance. Consequently, the screening energy stays independent of the kinetic energy of the colliding system and is given by $U_{\mathrm{e}}=Z_{1} Z_{2} e^{2} / D_{\mathrm{s}}$.

The experimental investigations of the last decade were focused on gas target studies [3] which, however, can include only the bound electron contribution to the screening energy. The experimental and theoretical values for $U_{\mathrm{e}}$ were found to be in reasonable agreement; discrepancies in some cases could be removed by means of corrections of the projectile stopping power values [4] or a better understanding of the reaction mechanism in the vicinity of the reaction threshold [5]. From both $\mathrm{d}+\mathrm{d}$ fusion reactions ${ }^{2} \mathrm{H}(\mathrm{d}, \mathrm{p})^{3} \mathrm{H}$ and ${ }^{2} \mathrm{H}(\mathrm{d}, \mathrm{n})^{3} \mathrm{He}$, only the former could so far be experimentally investigated at projectile energies low enough (below $10 \mathrm{keV}$ ) to observe the electron screening effect. The value of the screening energy was determined in the experiment using a gas target [6] to $(25 \pm 5) \mathrm{eV}$ which is very close to the theoretical value of $20 \mathrm{eV}$ [7].

However, an astrophysically relevant contribution for dense plasmas to the screening energy arising from unbound, free electrons has not been investigated experimentally so far. The application of metal hydrides as targets provides a unique possibility to study this contribution in the laboratory. A possible disadvantage of this method are many-body effects characteristic for dense matter resulting from internuclear correlation processes; but, due to trapping deuterons by the crystal lattice, these effects can be neglected [8].

In the present work we have studied the screening effect in both $\mathrm{d}+\mathrm{d}$ fusion reactions on three different deuteron-implanted metallic targets ( $\mathrm{Al}, \mathrm{Zr}$ and $\mathrm{Ta}$ ) in the deuteron energy range between 5 and $60 \mathrm{keV}$ [9]. We will show that the screening effect in metallic environments is much stronger than that for atomic or molecular gas targets and significantly stronger than the appropriate theoretical expectations. Due to the exponential behavior of relation (1), the increase of the screening energy can cause a drastic enhancement of the nuclear cross-section, especially in the case of the strong coupled plasma where the average Coulomb potential energy of ions exceeds their thermal energy.

Experimental set-up. - The experiment has been performed at a cascade accelerator with a highly stabilized $60 \mathrm{kV}$ power supply. The high voltage corresponding to the beam energy was measured by a precise voltage divider with an accuracy better than $1 \mathrm{~V}$. Beams of $\mathrm{D}^{+}$and $\mathrm{D}_{2}^{+}$ions produced in a high-frequency ion source were magnetically analyzed, focused and additionally deflected by about 10 degrees by a magnetic steerer located directly in front of the target chamber in order to remove neutral particles. Furthermore, a liquidnitrogen cooled $\mathrm{Cu}$ tube and a plate placed in the beam line and in the target chamber, respectively, were applied to avoid deposits on the target. The long-term energy stability was about $10 \mathrm{eV}$. The beam was focused on the target into a spot of about $1 \mathrm{~cm}$ in diameter. All charged particles $\left({ }^{3} \mathrm{He},{ }^{3} \mathrm{H}, \mathrm{p}\right)$ from $\mathrm{d}+\mathrm{d}$ reactions were detected with four $100 \mathrm{~mm}^{2}$ PIPSdetectors fixed at laboratory angles of $90^{\circ}, 110^{\circ}, 130^{\circ}$ and $150^{\circ}$ with respect to the beam at $10 \mathrm{~cm}$ distance from the target. Al foils of thickness $150 \mu \mathrm{g} / \mathrm{cm}^{2}$ in front of the detectors prevented elastically scattered deuterons from entering the detectors. The charge collected 


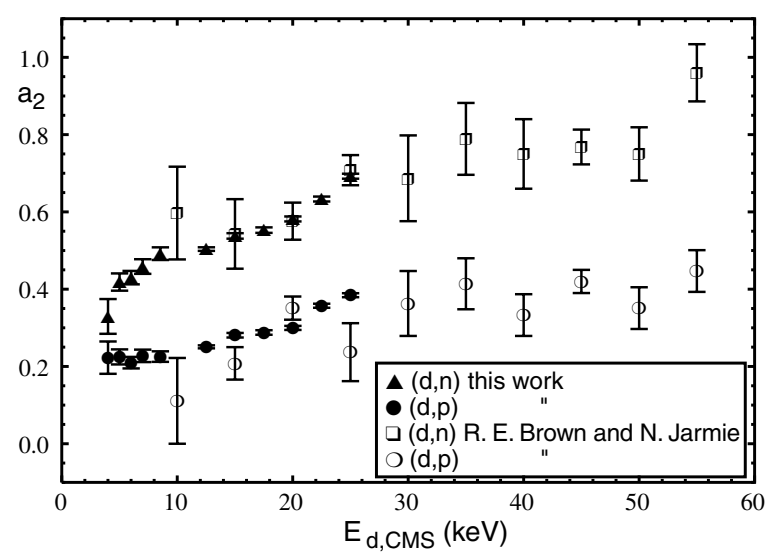

Fig. 1 - Anisotropy coefficient for the $(\mathrm{d}, \mathrm{p})$ and $(\mathrm{d}, \mathrm{n})$ reactions.

was determined via the measurement of the electric current on the target holder which was isolated from the target chamber. A negative voltage of $100 \mathrm{~V}$ was applied to a surrounding cylinder box for suppression of secondary electrons. The targets being thick $\mathrm{Al}, \mathrm{Zr}$ and $\mathrm{Ta}$ foils were implanted up to the saturation level close to the chemical stoichiometry using a $40 \mathrm{keV}$ deuteron beam. The implantation process was monitored by the proton yield of the ${ }^{2} \mathrm{H}(\mathrm{d}, \mathrm{p}){ }^{3} \mathrm{H}$ reaction. For details refer to [10].

Angular distribution. - The angular distributions of the ${ }^{2} \mathrm{H}(\mathrm{d}, \mathrm{p})^{3} \mathrm{H}$ and ${ }^{2} \mathrm{H}(\mathrm{d}, \mathrm{n})^{3} \mathrm{He}$ reactions are expected to be anisotropic even at the lowest projectile energies due to a relatively large $p$-wave component in the entrance channel. In the present work an angular distribution of a nuclear reaction could be measured for the first time at a beam energy as low as $5 \mathrm{keV}$. Neglecting an $L=2$ contribution, the angular distribution being symmetric with respect to $90^{\circ}$ can be parametrized as $A(\theta)=1+a_{2} \cos ^{2}(\theta)$. Our results, presented in fig. 1 , are in the overlapping energy region in good agreement with measurements of Brown and Jarmie [11]. The energy dependence of the parameter $a_{2}$ for both reactions recommended in ref. [11] could be confirmed by our results at extremely low energies. We observe no target material dependence of the angular distribution. This means that the screening effect which becomes visible for beam energies below $25 \mathrm{keV}$ should be equal for both entrance wave function contributions $L=0$ and $L=1$ and does not change the relative intensities of both contributions compared to the gas target experiments. A WKB calculation showed that the $L=1$ contribution is increased only by less than $5 \%$ relative to the $L=0$ contribution in the case of screening $\left(U_{\mathrm{e}}=300 \mathrm{eV}\right)$. So the parametrization given by Brown and Jarmie can also be applied to the present experiment.

Thick-target enhancement factor. - Apart from the angular distribution, we need information about the distribution of the implanted deuterons in the metallic targets for determining the total reaction rates and, consequently, the enhancement due to the screening effect. As was shown in [12], stable, homogeneously deuterized target layers can be obtained at the saturation level of implantation. Nevertheless, the stoichiometric ratio during longperiod measurements can vary slightly. Therefore, it was necessary to control on-line the deuteron density in the target. For this purpose the yield of the proton reaction channel has been continuously determined for all four detectors and stored together with the value of the beam charge collected in short-time periods. The thick-target angle-integrated reaction yield 


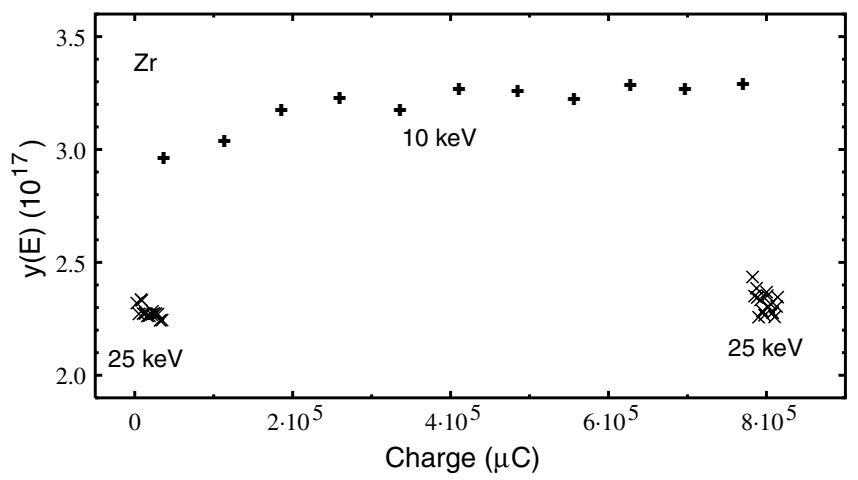

Fig. 2 - Reduced yields $y(E)$ over the charge collected at the target determined on-line for $E_{\text {lab }}=10$ and $25 \mathrm{keV}$.

including the screening effect can be written as

$$
Y_{\mathrm{scr}}(E)=\int_{0}^{E} \frac{n_{\mathrm{d}}}{\epsilon(E)} \sigma_{\mathrm{scr}}(E) \mathrm{d} E=\frac{n_{\mathrm{d}}}{n_{\mathrm{d}} A_{\mathrm{d}}+n_{\mathrm{m}} A_{\mathrm{m}}} \int_{0}^{E} \frac{1}{\sqrt{E}\left(E+U_{\mathrm{e}}\right)} S\left(E+U_{\mathrm{e}}\right) e^{-2 \pi \eta\left(E+U_{\mathrm{e}}\right)} \mathrm{d} E,
$$

where $A_{\mathrm{d}}, A_{\mathrm{m}}, n_{\mathrm{d}}$ and $n_{\mathrm{m}}$ are the stopping power coefficients and the number densities for the implanted deuterons and the metal atoms, respectively. The stopping power $\epsilon(E)=$ $-\mathrm{d} E / \mathrm{d} x=\left(n_{\mathrm{d}} A_{\mathrm{d}}+n_{\mathrm{m}} A_{\mathrm{m}}\right) \sqrt{E}$ is equated with that for protons at the same velocity [13] and includes the effect of implanted deuterons.

For the evaluation of the experimental thick-target results, it is appropriate to define the reduced yield function $y(E)$

$$
y(E)=\frac{Y_{\mathrm{scr}}(E)}{\int_{0}^{E} \frac{1}{E^{3 / 2}} S(E) e^{-2 \pi \eta(E)} \mathrm{d} E}=\frac{n_{\mathrm{d}}}{n_{\mathrm{d}} A_{\mathrm{d}}+n_{\mathrm{m}} A_{\mathrm{m}}} F(E) .
$$

$F(E)$ is the thick-target enhancement factor defined in analogy to the thin-target formula (relation (1)) as

$$
F(E)=\frac{Y_{\text {scr }}}{Y_{\text {bare }}}=\frac{\int_{0}^{E} \frac{1}{\sqrt{E}\left(E+U_{\mathrm{e}}\right)} S\left(E+U_{\mathrm{e}}\right) e^{-2 \pi \eta\left(E+U_{\mathrm{e}}\right)} \mathrm{d} E}{\int_{0}^{E} \frac{1}{E^{3 / 2}} S(E) e^{-2 \pi \eta(E)} \mathrm{d} E} .
$$

Due to electron screening, the function $y(E)$ will decrease with increasing $E$ and becomes the density ratio $n_{\mathrm{d}} /\left(n_{\mathrm{d}} A_{\mathrm{d}}+n_{\mathrm{m}} A_{\mathrm{m}}\right)$ for $E \gg U_{\mathrm{e}}$. In fig. 2 the on-line determined values of $y(E)$ at deuteron energy $10 \mathrm{keV}$ are compared with those achieved for our monitor measurements at $25 \mathrm{keV}$ (i.e. at a CMS energy $E_{0}=12.5 \mathrm{keV}$ ) performed before and after the $10 \mathrm{keV}$ measurements. The enhancement factor $F(E)$ can be determined from the observed jumps in the function $y(E)$ which occur between different energies but at equal deuteron density, as exemplified in fig. 2. Therefore the ratio $y_{\text {norm }}(E)=y(E) / y\left(E_{0}\right)$ is equal to the normalized enhancement factor $F_{\text {norm }}(E)=F(E) / F\left(E_{0}\right)$; this normalization makes the analysis independent of the deuteron density as well as of the stopping power values.

Results. - Our experimental results for the enhancement of the ${ }^{2} \mathrm{H}(\mathrm{d}, \mathrm{p})^{3} \mathrm{H}$ reaction yields investigated in the three metallic materials compared to the bare-nuclei case are presented in fig. 3. The experimental points, the uncertainties of which include both statistical and systematic errors (such as beam energy instability and changes in the deuteron density) are 


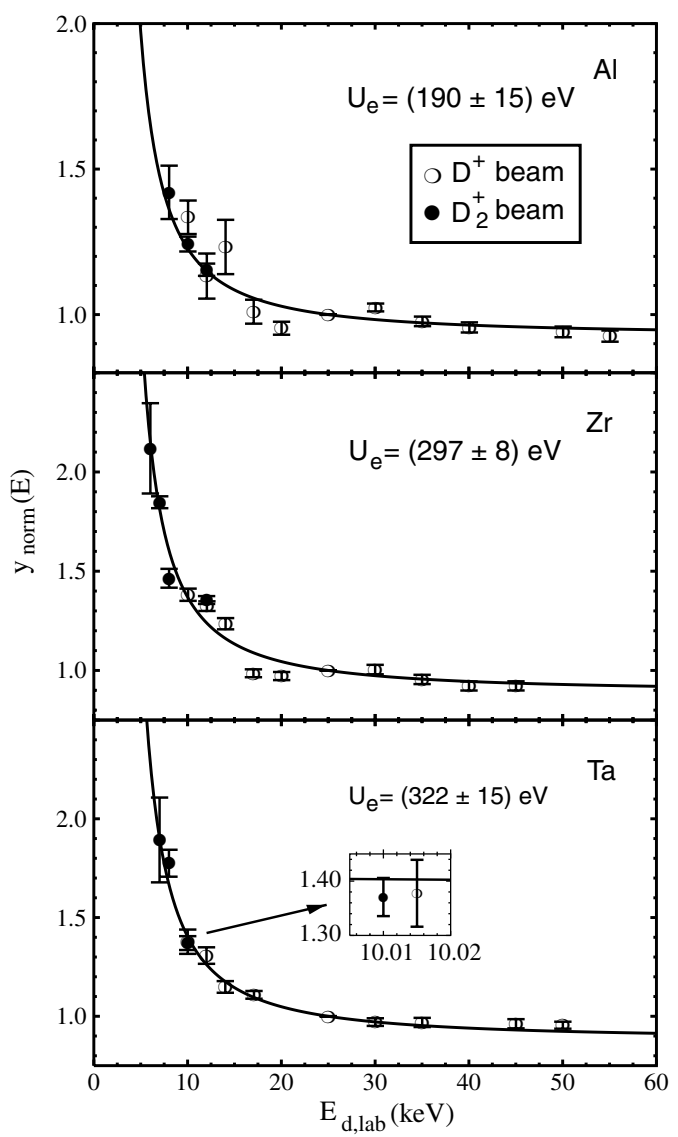

Fig. 3 - Enhancement of the thick-target yields for three different metals.

fitted by the function $F_{\text {norm }}(E)$ using only one parameter, the screening energy $U_{\mathrm{e}}$. Because of our normalization, the fitted curves take on unity at the deuteron energy $25 \mathrm{keV}$.

The ${ }^{2} \mathrm{H}(\mathrm{d}, \mathrm{n})^{3} \mathrm{He}$ reaction could simultaneously be observed by detecting the ${ }^{3} \mathrm{He}$ recoil nuclei. The ratio of the total reaction yields of both mirror reactions for the three metal targets $(\mathrm{Al}, \mathrm{Zr}, \mathrm{Ta})$ was found to be very close to one, which agrees with the parametrization of Brown and Jarmie [11] and with the experimental results achieved by Cecil et al. [14] and by Greife et al. [6].

The values of the screening energies $U_{\mathrm{e}}$ resulting from the fitting procedure described above are $(190 \pm 15) \mathrm{eV}$ for $\mathrm{Al},(297 \pm 8) \mathrm{eV}$ for $\mathrm{Zr}$ and $(322 \pm 15) \mathrm{eV}$ for Ta. They are about one order of magnitude larger than the value $U_{\mathrm{e}}=(25 \pm 5) \mathrm{eV}$ determined from a gas target experiment [6].

To exclude systematic errors as reason for such large values of the screening energies, we discuss in the following the possible sources of systematic uncertainties in detail.

For absolute cross-section measurements at sub-Coulomb energies an accurate knowledge of the beam energy is of crucial importance. Although the long-time high-voltage stability was better than $10 \mathrm{eV}$, the energy spread (FWHM) of the beam was much larger and amounted to about $150 \mathrm{eV}$. This value is characteristic for high-frequency ion sources and has been confirmed in opposite field measurements. An additional energy spread arises due to Coulomb explosion of the molecular $\mathrm{D}_{2}^{+}$beam in the target material [15]. For an incident energy below $12 \mathrm{keV}$ this energy spread is less than $100 \mathrm{eV}$. Assuming a Gaussian distribution for the beam 
energy and a constant $S$-factor over this energy interval, the weighted mean beam energy will be increased by about $10 \mathrm{eV}$ at $E_{\mathrm{d}, \mathrm{lab}}=5 \mathrm{keV}$ and by $7 \mathrm{eV}$ at $E_{\mathrm{d} \text {,lab }}=10 \mathrm{keV}$ which is comparable with the error of the fit for the screening energy and the high-voltage accuracy. Additionally, the beam energies determined by means of opposite field measurements were systematically lower by about $10 \mathrm{eV}$ than the value provided by the high-voltage divider. This effect can be explained by the electric potential drop arising in the plasma of the ion source. This correction has an opposite sign with respect to the correction due to the energy spread of the beam, so they cancel each other at the lowest projectile energies $(<12 \mathrm{keV})$ where we have used the molecular beam.

The next effect which could systematically influence our experimental results is the possibility of building up a thin carbon or oxide layer on the target surface. Since the deuteron density in such a layer is small compared to that achieved for the metal matrix, this effect reduces the measured reaction yield and corresponds to a smaller jump of the reduced yield $y(E)$, see fig. 2. Consequently, the experimental value for the screening energy would be too low. However, the off-line investigations of the target surfaces by means of Energy-Dispersive X-ray Micro Spectroscopy (EDX) did not show a significant contamination in the area of the beam spot. Possible deviations of the homogeneity of the deuteron distribution in the metal matrix can also lead to systematic errors. Due to the strong sputtering process of the metallic targets, it is likely that the deuteron density close to the target surface could be somewhat smaller. This, however, leads to a decrease of the determined screening energy in analogy to the case of a possible carbon-oxygen layer.

Since the last two effects could not be controlled on-line, the determined values for $U_{\mathrm{e}}$ should rather be treated as lower limits. A strong argument for the screening hypothesis is provided by the measured energy dependence of $y_{\text {norm }}$ (fig. 3 ) which can be fitted by $F_{\text {norm }}(E)$ using only one fit parameter, i.e. the screening energy $U_{\mathrm{e}}$. Additionally, the clearly observed target material dependence of $U_{\mathrm{e}}$ makes it quite unlikely that systematic errors might be responsible for the observed effects.

Discussion and conclusion. - The analysis of the experimental data in terms of the single parameter $U_{\mathrm{e}}$ does not exclude that this energy shift might be of an origin other than the electron screening. There are, however, no known effects specific for metals which could provide such a large energy push for the reacting nuclei. As discussed by You et al. [16], channelling of deuterons through the metal matrix or the interior adsorption effects can be described by an energy shift with a value much smaller than $10 \mathrm{eV}$, whereas screening from bound as well as from free electrons leads to significantly larger $U_{\mathrm{e}}$ values. The theoretical estimations for the electron screening energies have been performed mainly for deuterons implanted in palladium and in titanium, because of the interests related to cold-fusion experiments $[17,18]$. Both metals distinguish themselves by large diffusion coefficients of deuterons in the metal matrix. Zirconium also possesses a similar feature. The theoretical value of the screening energy calculated for palladium as the difference between the binding energies of an $\alpha$-particle and two deuterons [19] reaches only the value of $80 \mathrm{eV}$ which is a factor 4 less than the experimental value for zirconium and tantalum and a factor 2.5 less than the value for aluminium. This kind of estimation can be treated as an upper limit for Born-Oppenheimer-like calculations which, by definition, can include only static effects (the ion velocity converges to zero). A similar value has been determined by Ichimaru [8] using screening potentials obtained from the dispersion relation of the dielectric constant of the palladium deuteride. Such large discrepancies between theoretical and experimental values can indicate that the description of the electron screening should go beyond the static Born-Oppenheimer limit to include possible collective or dynamical effects for metallic electrons. One such approach is 
the time-dependent Hartree-Fock approximation [20]. In the frame of this method it could recently be shown that the screening energy of the system $d+D_{2}$ (a gas target) depends on the motion direction of the incident deuteron with respect to the symmetry axis of the target molecule $\mathrm{D}_{2}$. Similar calculations for metal hydrides have not been carried out so far because a large number of electron configurations has to be included. Another possibility to go beyond the Born-Oppenheimer approximation was presented by Federovich [21]. His method is based on the simultaneous solution of the Poisson and Schrödinger equations in the approximation of slow ( $s$-wave) electrons. He could show that the resulting screening potential between two reacting deuterons embedded in a free-electron sea becomes negative (attractive) at distances of about one Bohr radius. This effect could not be achieved in any other calculation.

In conclusion, we have determined the electron screening energies for $\mathrm{d}+\mathrm{d}$ fusion reactions in metallic environments. The large values of $U_{\mathrm{e}}$ and its target material dependence are theoretically not understood at present, which should be a challenge for future theoretical as well as experimental studies. On the one hand, the observed enhancement of the electron screening in metal targets can, in tendency, explain the small neutron production rates observed in the cold-fusion experiment of Jones [18]. According to calculations of Hora et al. [22], the corresponding value of the screening energy should amount to about $470 \mathrm{eV}$. On the other hand, larger values of the screening energies imply an increase of the reaction rates in astrophysical strong coupled plasmas. For weak coupled plasmas [23], including the Sun, this enhancement, although weaker, can also be of importance.

\section{REFERENCES}

[1] Salpeter E. E., Aust. J. Phys., 7 (1954) 373.

[2] Assenbaum H. J., Langanke K. and Rolfs C., Z. Phys. A, 327 (1987) 461.

[3] Rolfs C. and Somorjai E., Nucl. Instrum. Methods B, 99 (1995) 297.

[4] Langanke K., Shoppa T. D., Barnes C. A. and Rolfs C., Phys. Lett. B, 369 (1996) 211.

[5] Czerski K., Huke A., Bucka H., Heide P., Ruprecht G. and Unrau B., Phys. Rev. C, 55 (1997) 1517.

[6] Greife U. et al., Z. Phys. A, 351 (1995) 107.

[7] Shoppa T. D., Koonin S. E., Langanke K. and Seki R., Phys. Rev. C, 48 (1993) 837.

[8] IChimaru S., Rev. Mod. Phys., 65 (1993) 252.

[9] See also Czerski K., Huke A., Heide P., Hoeft M. and Ruprecht G., Nuclei in the Cosmos V, Proceedings of the International Symposium on Nuclear Astrophysics, July 6-11, 1998 Volos, Greece, edited by N. Prantzos and S. Harissopulos (Editions Frontières) p. 152.

[10] Huke A., PhD thesis, Technische Universität Berlin, 2001.

[11] Brown R. E. and Jarmie N., Phys. Rev. C, 41 (1990) 1391.

[12] Myers S. M. et al., Phys. Rev. B, 43 (1991) 9503.

[13] Anderson H. and Ziegler J. F., The Stopping and Ranges of Ions in Matter, Vol. 3 (Pergamon Press, New York) 1977.

[14] Cecil F. E., Liu H., Yan J. S. and Hale G. M., Phys. Rev. C, 47 (1993) 1178.

[15] Vogler M. and Meierjohann B., Z. Phys. A, 283 (1977) 11.

[16] You J. H., Cheng F. H., Cheng F. Z. and Huang F. H., Phys. Rev. B, 43 (1991) 7293.

[17] Fleischmann M., Pons S. and Hawkins M., J. Electroanal. Chem., 261 (1989) 301.

[18] Jones S. E. et al., Nature, 338 (1989) 737.

[19] Legett A. J. and Baym G., Phys. Rev. Lett., 63 (1989) 191.

[20] Shoppa T. D. et al., Nucl. Phys. A, 605 (1996) 387.

[21] Federovich G. V., Phys. Lett. A, 164149 (1992.)

[22] Hora H. et al., Phys. Lett. A, 175 (1993) 138.

[23] Brown L. S. and Sawyer R. F., Rev. Mod. Phys., 69 (1997) 411. 